Circadian preference and sleep timing from childhood to adolescence in relation to genetic variants from a genome-wide association study

Merikanto, llona

2018-10

Merikanto , I, Lahti , J , Kuula , L , Heinonen , K, Räikkönen , K, Andersson , S , Strandberg, T \& Pesonen, A-K 2018 , ' Circadian preference and sleep timing from childhood to adolescence in relation to genetic variants from a genome-wide association study ' , Sleep Medicine , vol. 50 , pp. 36-41 . https://doi.org/10.1016/j.sleep.2018.04.015

http://hdl.handle.net/10138/247828

https://doi.org/10.1016/j.sleep.2018.04.015

publishedVersion

Downloaded from Helda, University of Helsinki institutional repository.

This is an electronic reprint of the original article.

This reprint may differ from the original in pagination and typographic detail.

Please cite the original version. 
Original Article

\section{Circadian preference and sleep timing from childhood to adolescence in relation to genetic variants from a genome-wide association study}

Ilona Merikanto a, b, c, *, Jari Lahti a , Liisa Kuula ${ }^{a}$, Kati Heinonen a, Katri Räikkönen a, Sture Andersson ${ }^{\mathrm{d}}$, Timo Strandberg ${ }^{\mathrm{e}, \mathrm{f}}$, Anu-Katriina Pesonen ${ }^{\mathrm{a}}$

a Department of Psychology and Logopedics, Faculty of Medicine, University of Helsinki, Helsinki, Finland

${ }^{\mathrm{b}}$ National Institute for Health and Welfare, Helsinki, Finland

c Orton Orthopaedics Hospital, Helsinki, Finland

d Children's Hospital, University of Helsinki, and Helsinki University Hospital, Helsinki, Finland

e University of Helsinki and Helsinki University Hospital, Helsinki, Finland

${ }^{\mathrm{f}}$ University of Oulu, Center for Life Course Health Research, Oulu, Finland

\section{A R T I C L E I N F O}

\section{Article history:}

Received 23 February 2018

Received in revised form

19 April 2018

Accepted 23 April 2018

Available online 1 June 2018

\section{Keywords:}

Actigraphy

Chronotype

Circadian rhythm

Longitudinal

Polygenic risk score

Sleep midpoint

\begin{abstract}
A B S T R A C T
Objective: Recent genome-wide association studies (GWASs) have revealed new genetic variants behind self-reported individual circadian preference, a distinct biological trait that is fairly stable during adulthood. In this study we analyze whether these genetic variants associate with objectively measured sleep timing from childhood to adolescence, over a nine-year period, with self-reported circadian preference during late adolescence.

Methods: The participants $(N=100,61 \%$ girls) came from a community cohort from Finland born in 1998. Sleep midpoint was measured with actigraphy at 8,12 and 17 years. Circadian preference was selfreported at the age of 17 years. Single nucleotide polymorphisms (SNPs) were extracted at 12 years of age from the Illumina OmniExpress Exome 1.2 bead array data. Weighted polygenic risk scores (PRSs) were calculated based on top SNPs from a recent GWAS for morningness-eveningness in an adult population. Results: The PRS for circadian preference towards morningness was associated with earlier sleep midpoint from childhood to adolescence. When the time points were analyzed separately, the association between genetic tendency towards morning preference and earlier sleep midpoint was strongest among the 17-year-olds. Furthermore, the shift towards later sleep rhythm from early to late adolescence was milder for those with a higher PRS for morning preference. PRS for morning preference was also associated with self-reported circadian preference towards morningness in late adolescence.

Conclusion: Our results suggest that genetic variants found for circadian preference in adults are already associated with objective sleep timing during childhood and adolescence, and predict individual developmental sleep trajectories from childhood onwards.
\end{abstract}

(c) 2018 Published by Elsevier B.V.

\section{Introduction}

Individual circadian rhythm governs the daily timing of behavior and physiological functions, such as sleep-wake rhythm, hormonal secretion, blood pressure, and body temperature [1]. The epidemiology of circadian preferences is a subject of interest as individuals with a circadian preference towards eveningness have a higher risk for multiple health problems as compared to morning-oriented

\footnotetext{
* Corresponding author. Department of Psychology and Logopedics, Faculty of Medicine, University of Helsinki, P.O. Box 9, University of Helsinki 00014, Helsinki, Finland.

E-mail address: anukatriina.pesonen@helsinki.fi (I. Merikanto).
}

individuals [2-13]. Although there is considerable variation between individuals in their circadian rhythm, the intra-individual stability in adulthood is high [14], and falls on the continuum of early and late circadian preferences [15]. However, over the course of a lifetime circadian rhythmicity shows age-related tendencies: during childhood the circadian timing is generally more morning-oriented and it becomes progressively more evening-oriented during adolescence until the peak in eveningness preference is reached in late adolescence [16]. Finally, a shift towards morningness is seen in old age [14]. We have previously shown that 17 -year-old adolescents with a selfreported eveningness preference already had a later objectively measured sleep timing at the age of eight years [17]. While the later sleep rhythm showed significant continuity from eight to 17 years, the 
most marked change towards later timing of sleep was seen between the ages of 12 and 17 years [17].

An individual's circadian rhythms are influenced by environmental cues, especially exposure to daylight [18], and by genetic factors, as indicated by three recent genome-wide association studies (GWASs) on adult chronotype [19-21]. From several genes associated with circadian rhythm, all three studies associated the same four genes to circadian preference, PER2, RGS16, AK5, and FBXL13 [19-21], of which PER2, FBXL13, and RGS16 have a known role in the circadian regulation [22-28]. However, the validity of these findings has not been tested at earlier ages or with an objective measurement of sleep with actigraphy.

Accordingly, we set out to analyze the effect of single nucleotide polymorphisms (SNPs) significantly associated with self-reported circadian preference by associating weighted polygenic risk score (PRS) with objectively measured sleep midpoint across three time points from childhood to adolescence, and with a self-report of circadian preference at the age of 17 years. PRS based on GWAS results have been widely used recently and increase the power to detect associations [29]. For the PRS for circadian preference, we used the findings from the most recent and largest of the three GWAS studies, which is based on the UK Biobank study data on British adults [19]. We hypothesized that genetic variants associated with self-reported circadian preference in GWAS influence the actualized objective sleep timing from childhood to adolescence and also the subjective circadian preference in late adolescence. We base these assumptions on our observation of a significant continuity in objective sleep timing from childhood to adolescence, that reflected the individual's circadian preference [17]. However, we also expected to see differences in the strength of the genetic effect on sleep midpoint by age, as the period from childhood to adolescence encompasses a progressive shift in circadian rhythm towards eveningness [16].

\section{Material and methods}

\subsection{Participants}

The data in this study come from an urban community-based cohort comprising initially 1049 healthy singletons born between March and November 1998 in Helsinki, Finland. The first two sleep follow-ups were conducted during the years 2006 and 2009-2011, at eight (T1) and 12 (T2) years of age (on average). The details of the cohort are described in more detail in previous reports [30-32]. In years 2014-2015, all the cohort members who participated in the previous follow-up at age 12 years, and who lived within the $30-\mathrm{km}$ radius from Helsinki ( $N=279,77.1 \%$ of the participants of the previous follow-up) were invited to a follow-up. Of them, 197 (70.6\%) participated at the age of 17 (T3). Fig. 1 illustrates the study design. The analytic sample $(N=100$, girls $=61$, boys $=39)$ consisted of those with complete information on (1) each actigraph-based sleep measurement at $\mathrm{T} 1$ (mean age $=8.1$, standard deviation $(\mathrm{SD})=0.3$ years), $\mathrm{T} 2$ (mean age $=11.8$, $\mathrm{SD}=0.5$ years) and $\mathrm{T} 3$ (mean age $=16.9, \mathrm{SD}=0.1$ years) for at least four nights, (2) self-reported circadian preference at T3, and (3) genetic samples extracted at T2. The Ethics Committee for Children and Adolescents' Diseases and Psychiatry at the Helsinki University Central Hospital approved the study protocol. All participants and their parents gave their written informed consent.

\subsection{Objective measure of circadian timing by actigraphy}

The habitual sleep duration was measured by actigraphs worn on the non-dominant wrist at three time points across nine years: at T1 (mean nights $=7.0, \mathrm{SD}=1.3$ ), $\mathrm{T} 2$ (mean nights $=7.9, \mathrm{SD}=2.1$ ), and T3 (mean nights $=8.3, \mathrm{SD}=2.0$ ) (T1 Actiwatch AW4, T2 and T3 Actiwatch AW7, Cambridge Neurotechnology Ltd., UK).The child/adolescent participants were instructed to report the wake-up time and bedtime by pressing an event marker button in the actigraph device as well as by keeping a sleep log. Temporary pauses in the actigraph usage were also instructed to be marked into the sleep log, including, eg, periods of illnesses, or travels. The following reasons led to exclusion of a particular night from the participant's sleep data: (1) the actigraph was not in use; (2) information on bedtimes was missing; (3) the data on sleep log reported bedtime indicated that the child/adolescent was already asleep (suggesting that the bedtime was incorrectly reported); (4) information on waking time was missing and the activity pattern was unclear; or (5) there was a change in everyday life due to, eg, illness or travel, or alcohol use (adolescents). Sleep midpoint at each time point was determined from the assumed sleep duration as the half of the time passed in sleep and also calculated separately for weekdays and weekends. Following Roenneberg et al. [16], we also calculated the sleep deprivation corrected sleep midpoint for weekends at each time point with the following algorithm: corrected weekend sleep midpoint $=$ weekend sleep midpoint $-0.5 \times$ (mean sleep duration on weekend- $5 \times$ mean sleep duration on weekdays $+2 \times$ mean sleep duration on the weekend)/7) [16]. Corrected sleep midpoint for weekend correlated with the uncorrected weekend sleep midpoint at T1 $\left(r=0.95, p=1.2 \times 10^{-49}\right)$, at T2 $\left(r=0.94, p=1.8 \times 10^{-43}\right)$ and at T3 $\left(r=0.98, p=1.2 \times 10^{-71}\right)$.

\subsection{Subjective circadian preference assessment}

To replicate the design in the GWAS by Jones and colleagues [19], we used the same one question as they did for self-reported chronotype from the Horne-Östberg Morningness-Eveningness Questionnaire (MEQ item 19) to define circadian preference. This item correlated significantly with the full MEQ $(r=0.41$, $p=0.0004$ ) [33] in our sample. The question asks individuals to estimate their circadian preference as either $1=$ Definitely a
$\mathrm{T} 1$

Collected during 2006

Mean age $=8.1, S D=0.3$ years $\mathrm{N}=100$
T2

Collected during 2009-2011,

Mean age $=11.8, \mathrm{SD}=0.5$ years $\mathrm{N}=100$
T3

Collected during 2014-2015,

Mean age $=16.9, \mathrm{SD}=0.1$ years $\mathrm{N}=100$

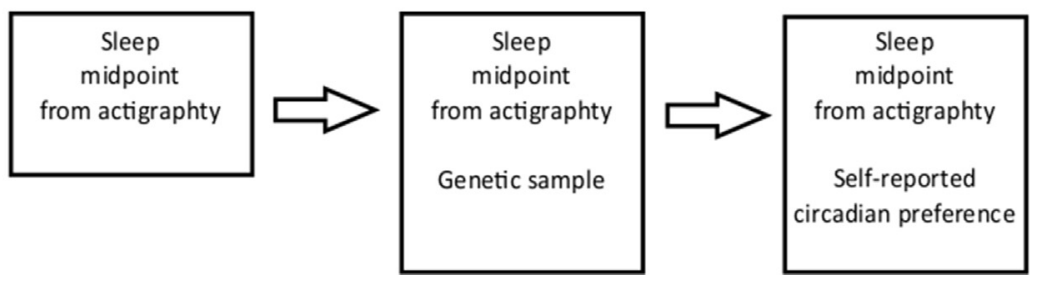

Fig. 1. Longitudinal study design. 
'morning' person, 2 = More a 'morning' than an 'evening' person, $3=$ More an 'evening' than a 'morning' person, or $4=$ Definitely an 'evening' person. Similarly to Jones et al., [19], we grouped the definite and moderate morning preferences together (answers 1 and 2) and did the same for definite and moderate evening preferences (answers 3 and 4) thus forming a dichotomous circadian preference for morningness-eveningness. Our sample yielded 20 participants with morning preference (of which 13 were girls, $65 \%$ ) and 80 participants with evening preference (of which 48 were girls, $60 \%$ ). We also used the full MEQ as a continuous variable in relation to PRS. The full MEQ was available for 71 participants with data on sleep midpoint at each age point.

\subsection{Genotyping, SNP selection and calculation of the weighted PRS}

DNA was extracted from blood (22\%) and saliva samples (78\%) collected at the 2009-2011 follow-up, and the genotyping was performed with the Illumina OmniExpress Exome 1.2 bead chip at the Tartu University, Estonia, in September 2014, according to the standard protocols. Genomic coverage was extended by imputation using the 1000 Genomes Phase I integrated variant set (v3/April 2012; NCBI build 37/hg19) as the reference sample and IMPUTE2 software. Before imputing, the following quality-control filters were applied: SNP clustering probability for each genotype $>95 \%$, Call rate $>95 \%$ individuals and markers (99\% for markers with minor allele frequency (MAF) $<5 \%$ ), MAF $>1 \%$, Hardy-Weinberg equilibrium (HWE) $p>1 \times 10^{-6}$. Moreover, heterozygosity, sex check, and relatedness checks were performed, and any discrepancies removed $(N=2)$.

Of this data, we set out to extract 13 SNPs with $p<5 \times 10^{-8}$ in GWAS by Jones et al., for self-reported chronotype, based on data from 128,266 British adults, aged between 37 and 73 years, from the UK Biobank study and replicated in 89,283 23andMe participants [19]. All of these SNPSs were not available in our sample, with rs76899638 missing (a detailed list of the available SNPs in our sample are presented in Table 1). The extracted SNPs for genetic analyses thus included 12 SNPs from the GWAS by Jones et al. for morning preference [19]. Of the extracted SNPs, the variant rs516134 was filtered out from the PRS calculation as it had lower MAF than 5\% (see Table 1 for MAF results). PRS was calculated by multiplying the number of effect alleles at each $\operatorname{SNP}(0,1,2)$ with a logarithmic transformation of the corresponding odds ratio for morning preference reported in the GWAS [19] (odds ratios for SNPs are presented in Table 1) and then summing the scores across the SNPs.

\subsection{Statistical analysis}

To calculate differences in sleep midpoint at T1, T2, and T3 by circadian preference we used one-way ANOVA for morning/evening preference assessed with item 19 of the full MEQ [34] and correlation analyses for continuous circadian preference assessed with full MEQ. We used mixed model analyses to study the association between PRS and sleep midpoint across time separately for weekdays and weekends over the period of nine years. We also studied whether the sleep midpoint developed differently across the time points depending on PRS by entering an interaction term 'PRS $\times$ time' in the mixed model. To study whether any of the associations were moderated by sex, we entered an interaction term 'PRS $\times$ sex' to mixed models. If the main PRS effect or the interaction was significant, we analyzed the association between PRS and each sleep midpoint, or the change in midpoint from T1 to T2 and from T2 to T3, with a generalized linear model (GLM). The association between PRS and self-reported circadian preference was also analyzed with GLM, as were the associations between individual SNPs and each sleep midpoint measurements. All mixed model and GLM analyses were adjusted for age and sex.

\section{Results}

\subsection{Objective sleep midpoint by self-reported circadian preference} from $T 1$ to $T 3$

Similarly to previously reported results [17], those with a selfreported evening preference had significantly later weekend sleep midpoint from T1 years to T3 than those with morning preference (at $\mathrm{T} 1 p=0.04$, at T2 $p=0.01$, and at T3 $p=0.02$ ). Also the continuous full MEQ score correlated with weekday and weekend sleep midpoint at T2 and T3 (at T1 $r_{\text {weekday }}=-0.05, p=0.7$ and $r_{\text {weekend }}=-0.2$, $p=0.07 ;$ at $\mathrm{T} 2 \mathrm{r}_{\text {weekday }}=-0.2, p=0.04$ and $\mathrm{r}_{\text {weekend }}=-0.3, p=0.03$; at T3 $r_{\text {weekday }}=-0.3, p=0.006$ and $r_{\text {weekend }}=-0.3, p=0.02$ ). At T1, the difference in weekend midpoint between the morning and evening preference types was $21 \mathrm{~min}$, at T2 it was $31 \mathrm{~min}$, and at T3 it was $1 \mathrm{~h}$ and $31 \mathrm{~min}$ (Table 2).

\subsection{PRS and sleep midpoint measured with actigraphy}

PRS for morning preference was associated with earlier sleep midpoint over time in mixed models for weekends $(B=-3.2,95 \%$ $\mathrm{CI}=-6.2$ to $-0.09, p=0.04)$, but not for weekdays $(\mathrm{B}=-2.2,95 \%$ $\mathrm{CI}=-4.5$ to $0.05, p=0.06$ ). Next, we performed mixed model analysis with 'time $\times$ PRS' interaction term to see whether sleep midpoint developed differently across the time points depending on the weighted PRS. This interaction was significant both for weekdays $(p=0.003)$ and for weekends $(p=0.04)$. No significant results were yielded for the 'gender $\times$ PRS' interaction terms $(p \geq 0.3)$. When we examined the associations separately at each time point, no statistically significant associations between the PRS for morning preference and weekend sleep midpoint was found (all $p \geq 0.08$; Table 3 ). PRS for morning preference was associated with earlier sleep midpoint in weekdays at T3 ( $p=0.006$, Table 3$)$.

As the 'time $\times$ PRS' interaction was significant, we examined the change in midpoints from T1 to T2 and from T2 to T3 in relation to PRS. The change in weekday midpoint towards eveningness from T2 to T3 was significantly lower among those with higher PRS for morning preference $(\mathrm{B}=-6.9,95 \% \mathrm{CI}=-11.9$ to $-2.0, p=0.006$, see Fig. 2). The change in weekend midpoint from $\mathrm{T} 1$ to $\mathrm{T} 2(\mathrm{~B}=1.2$, $95 \% \mathrm{CI}=-2.5$ to $4.9, p=0.5$ ) or from $\mathrm{T} 2$ to $\mathrm{T} 3$ ( $\mathrm{B}=-3.1,95 \%$ $\mathrm{CI}=-13.9$ to $7.7, p=0.6)$ and the change in weekday midpoint from $\mathrm{T} 1$ to $\mathrm{T} 2(2.3,95 \% \mathrm{CI}=-0.6$ to $5.1, p=0.1)$ were not significantly associated with the PRS.

Earlier sleep deprivation corrected weekend sleep midpoint was also associated with PRS for morning preference over time in the mixed model $(\mathrm{B}=-3.5,95 \% \mathrm{CI}=-6.5$ to $-0.5, p=0.02)$. When we examined the associations separately at each time point, no statistically significant associations between the PRS for morning preference and corrected weekend sleep midpoint was found (all $p \geq 0.07$; Table 3).

\subsection{Individual SNPS and midpoint of sleep}

Four SNPs from the 11 selected SNPs from the GWAS study by Jones et al., [19] were associated significantly with sleep midpoint during childhood or adolescence. The effect alleles for morning preference of the rs75804782 (T) and rs372229746 (G) associated at T1 with earlier sleep midpoint both during weekdays (for rs75804782 $p=0.04$ and for rs372229746 $p=0.03$ ) and weekends (for rs75804782 $p=0.01$ and for rs372229746 $p=0.04$ ). The effect allele for morning preference of the rs9961653 (T) was associated with earlier weekend midpoint at T1 $(p=0.04)$ as well as later weekday sleep midpoint at T3 $(p=0.03)$. The effect allele for morning preference of the rs11162296 (G) was associated with later rather than earlier weekday sleep midpoint at T2 $(p=0.04)$. The 
Table 1

The selected SNPs for genetic analyses from the GWAS [19] for morningness-eveningness.

\begin{tabular}{|c|c|c|c|c|c|c|c|c|c|c|c|c|c|}
\hline SNP & Chr:Pos & $\mathrm{A} 1 / \mathrm{A} 2$ & $\begin{array}{l}\text { Effect } \\
\text { allele }\end{array}$ & $\begin{array}{l}\text { Effect allele } \\
\text { frequency } \\
\text { in the original } \\
\text { GWAS }\end{array}$ & $\begin{array}{l}\text { Imputation } \\
\text { quality }\end{array}$ & HWE & $\begin{array}{l}\text { MAF for } \\
\text { minor } \\
\text { allele }\end{array}$ & Missing & $\begin{array}{l}\text { OR for } \\
\text { morning } \\
\text { preference } \\
\text { in the } \\
\text { original } \\
\text { GWAS }\end{array}$ & $\begin{array}{l}p \text { for OR in } \\
\text { the original } \\
\text { GWAS }\end{array}$ & $\begin{array}{l}\text { Beta for } \\
\text { continuous } \\
\text { chronotype } \\
\text { in the } \\
\text { original } \\
\text { GWAS }\end{array}$ & $\begin{array}{l}p \text { for Beta } \\
\text { in the } \\
\text { original } \\
\text { GWAS }\end{array}$ & $\begin{array}{l}\text { Nearest gene } \\
\text { in the } \\
\text { original GWAS }\end{array}$ \\
\hline rs516134 & $1: 182,553,693$ & $\mathrm{C} / \mathrm{T}$ & C & 0.03 & 1 & 1 & 0.01831 & 0 & 1.21 & $3 \times 10^{-12}$ & 0.081 & $9 \times 10^{-13}$ & RGS16 \\
\hline rs11162296 & $1: 77,700,196$ & $\mathrm{C} / \mathrm{G}$ & G & 0.84 & 0.99952 & 0.0738294 & 0.20696 & 0 & 0.93 & $1 \times 10^{-12}$ & -0.037 & $2 \times 10^{-12}$ & PIGK, AK5 \\
\hline rs77641763 & $9: 140,265,782$ & $\mathrm{~T} / \mathrm{C}$ & C & 0.88 & 0.99551 & 0.3279505 & 0.20638 & 0 & 1.07 & $7 \times 10^{-9}$ & 0.039 & $5 \times 10^{-11}$ & EXD3 \\
\hline rs1075265 & $2: 54,354,927$ & $\mathrm{C} / \mathrm{G}$ & C & 0.48 & 0.9963 & 0.2625148 & 0.39273 & 0 & 0.95 & $4 \times 10^{-8}$ & -0.025 & $2 \times 10^{-10}$ & PSME4, ACYP2 \\
\hline rs72720396 & $1: 91,191,582$ & $\mathrm{G} / \mathrm{A}$ & A & 0.77 & 0.99612 & 0.2122820 & 0.18157 & 0 & 0.95 & $3 \times 10^{-8}$ & -0.025 & $1 \times 10^{-7}$ & CALB1 \\
\hline rs75804782 & $2: 239,316,043$ & $\mathrm{C} / \mathrm{T}$ & $\mathrm{T}$ & 0.88 & 0.98898 & 0.0527485 & 0.17051 & 0 & 1.09 & $4 \times 10^{-10}$ & 0.030 & $3 \times 10^{-7}$ & PER2 \\
\hline rs10157197 & $1: 150,250,636$ & $\mathrm{~A} / \mathrm{G}$ & G & 0.6 & 1 & 0.2072574 & 0.36338 & 0 & 1.05 & $5 \times 10^{-7}$ & 0.025 & $1 \times 10^{-9}$ & PRPF3, TARS2 \\
\hline rs372229746 & $7: 102,158,815$ & $\mathrm{~A} / \mathrm{G}$ & G & 0.55 & 0.67764 & 0.3257922 & 0.42998 & 0 & 1.06 & $7 \times 10^{-7}$ & 0.028 & $4 \times 10^{-9}$ & ORAI2 \\
\hline rs9961653 & $18: 56,767,671$ & $\mathrm{~T} / \mathrm{C}$ & $\mathrm{T}$ & 0.42 & 1 & 0.9125697 & 0.40282 & 0 & 1.04 & $1 \times 10^{-6}$ & 0.023 & $1 \times 10^{-8}$ & $\begin{array}{l}\text { RAX, CPLX4, } \\
\text { LMAN1 }\end{array}$ \\
\hline rs2050122 & $1: 19,989,205$ & $\mathrm{~T} / \mathrm{C}$ & $\mathrm{T}$ & 0.2 & 0.99738 & 1 & 0.17679 & 0 & 1.06 & $3 \times 10^{-6}$ & 0.028 & $2 \times 10^{-8}$ & HTR6 \\
\hline rs70944707 & $2: 24,257,444$ & $\mathrm{C} / \mathrm{CT}$ & $\mathrm{C}$ & 0.23 & 0.87012 & 0.8795015 & 0.28369 & 0 & 1.05 & $2 \times 10^{-5}$ & 0.030 & $3 \times 10^{-8}$ & FKBP1B \\
\hline rs12140153 & $1: 62,579,891$ & $\mathrm{~T} / \mathrm{G}$ & G & 0.9 & 1 & 1 & 0.078873 & 0 & 1.07 & $4 \times 10^{-6}$ & 0.039 & $7 \times 10^{-9}$ & INADL \\
\hline
\end{tabular}

For the analyses we used logarithmic transformation of the odds ratio (OR) when calculating PRSs.

A1, minor allele in the current sample data; A2, major allele in the current sample data; Chr, chromosome.

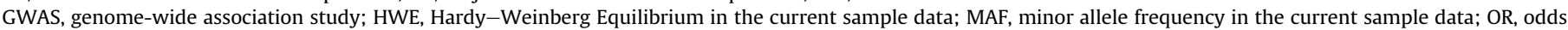
ratio (ie, effect size for genetic associations in GWAS for morning preference); Pos, position; SNP, single nucleotide polymorphism.

GLM results from the association between selected SNPs and each midpoint measurement are shown in Supplementary Table S1.

Similar to uncorrected weekend midpoint, earlier sleep deprivation corrected sleep midpoint was associated with the effect alleles for morning preference of the rs75804782 (allele $\mathrm{T}, \mathrm{B}=-9.9$, $95 \% \mathrm{CI}=-17.6$ to $2.3, p=0.01$ ), rs372229746 (allele $\mathrm{G}, \mathrm{B}=-10.9$, $95 \% \mathrm{CI}=-20.2$ to $1.7, p=0.02$ ) and rs9961653 (allele $\mathrm{T}, \mathrm{B}=-12.5$, $95 \% \mathrm{CI}=-23.0$ to $2.0, p=0.02$ ) at T1. The effect allele for morning preference of the rs12140153 (allele $\mathrm{G}, \mathrm{B}=18.8,95 \% \mathrm{CI}=1.5-36.1$, $p=0.03$ ) was associated with later rather than earlier corrected weekend sleep midpoint similar to weekday sleep midpoint at T2 (all other $p$-values at $\mathrm{T} 1 \geq 0.08, p$-values at $\mathrm{T} 2 \geq 0.06$, and $p$-values at $\mathrm{T} 3 \geq 0.2$ ).

\subsection{PRS and self-reported circadian preference}

PRS for morning preference was associated with self-reported circadian preference for morningness based on a single MEQ item ( $\mathrm{B}=-1.8,95 \% \mathrm{CI}=-3.4$ to $-0.1, p=0.03$, measured only at $\mathrm{T} 3$ ). Circadian preference assessed as continuous with the full MEQ was not associated with PRS for morning preference $(\mathrm{B}=-4.3,95 \%$ $\mathrm{CI}=-36.3$ to $27.6, p=0.8$, measured only at T3). The selected SNPs from the GWAS by Jones et al., [19] were not statistically significantly (all $p \geq 0.09$ ) associated with circadian preference when analyzed individually in GLM (results not shown).

Table 2

Mean number of nights and sleep midpoint at each age measurement point by selfreported circadian preference at age 17 years.

\begin{tabular}{lllll}
\hline Actigraph measurements & Morning & & Evening & $p$ \\
\cline { 2 - 2 } & Mean $\pm \mathrm{SD}$ & & Mean $\pm \mathrm{SD}$ & \\
\hline Number of nights, age 8 years & $7.0 \pm 1.4$ & & $7.0 \pm 1.3$ & 0.9 \\
Number of nights, age 12 years & $7.0 \pm 2.3$ & & $7.9 \pm 1.8$ & 0.9 \\
Number of nights, age 17 years & $8.8 \pm 1.9$ & & $8.2 \pm 1.6$ & 0.1 \\
Sleep midpoint weekdays, age 8 years & $2: 23 \pm 0: 34$ & & $2: 25 \pm 0: 34$ & 0.8 \\
Sleep midpoint weekend, age 8 years & $2: 36 \pm 0: 42$ & & $2: 57 \pm 0: 39$ & 0.04 \\
Sleep midpoint weekdays, age 12 years & $2: 48 \pm 0: 24$ & & $3: 02 \pm 0: 42$ & 0.2 \\
Sleep midpoint weekend, age 12 years & $3: 32 \pm 0: 39$ & & $4: 03 \pm 0: 47$ & 0.01 \\
Sleep midpoint weekdays, age 17 years & $3: 30 \pm 0: 48$ & & $4: 08 \pm 1: 30$ & 0.05 \\
Sleep midpoint weekend, age 17 years & $4: 29 \pm 0: 35$ & & $6: 00 \pm 2: 42$ & 0.02 \\
\hline
\end{tabular}

$\mathrm{SD}$, standard deviation; $p$ from one-way analysis of variance.

\section{Discussion}

Our study showed how genetic factors, previously associated with circadian preference only in adults, associated significantly with the objectively measured sleep timing from middle childhood to late adolescence in this longitudinal community cohort. As assumed on the basis of the original GWAS finding [19], the PRSs for morning preference were associated with earlier sleep midpoint, as averaged across the nine year follow-up period. The associations between the PRS and an earlier sleep midpoint were significant regarding both weekdays and weekends. As a further contribution, the study showed that the sleep midpoint trajectories across the nine-year follow-up were moderated by the PRS score. While it is well known that sleep rhythm shifts to later during adolescence [16], our study showed for the first time that the shift for later circadian rhythm from early to late adolescence (ie, from age $12-17$ years) was significantly milder among individuals with a high PRS for morning preference.

When the sleep midpoint was examined separately at each time point, the negative association between sleep midpoint on weekdays and PRS was statistically significant at age 17 years, and the negative association between sleep midpoint at weekends and PRS was approaching statistical significance at age eight years. When the sleep deprivation corrected weekend midpoint was used, the results were essentially the same. It might then be speculated that towards late adolescence, there is a decreasing parental authority involved in regulation of the sleep rhythm, and the genetic influence becomes more emphasized. It is also noteworthy that even at the age of eight years, the genetic influence plays a part especially on weekends, where children are able to sleep more freely.

The self-reported circadian preference, measured only at age 17 years in our study, was also associated with the PRS for morning preference, in line with the expectation. No significant association between PRS for morningness and full MEQ circadian preference was found. However, it must be remembered that the original GWAS is based on the single-item approach [19].

Four out of 11 selected SNPs from the GWAS [19] had a significant effect on sleep midpoint during childhood. The $T$ allele of the rs75804782 was associated with an earlier sleep midpoint at the age of eight years on weekdays and weekends. This variant locates near PER2 (Period Circadian Clock 2), which is one of the key regulator genes 
Table 3

Polygenic risk scores for morning preference in relation to sleep midpoint during adolescence in generalized linear model analysis.

\begin{tabular}{|c|c|c|c|c|c|c|}
\hline & Age 8 years & $p$ & Age 12 years & $p$ & Age 17 years & $p$ \\
\hline & $\mathrm{B}(95 \% \mathrm{CI})$ & & $\mathrm{B}(95 \% \mathrm{CI})$ & & $\mathrm{B}(95 \% \mathrm{CI})$ & \\
\hline Weekday midpoint & $-1.4(-3.8$ to 0.9$)$ & 0.2 & $1.0(-1.9$ to 3.8$)$ & 0.5 & $-6.1(-10.4$ to -1.8$)$ & 0.006 \\
\hline Weekend midpoint & $-2.6(-5.4$ to 0.3$)$ & 0.08 & $-0.5(-3.9$ to 2.9$)$ & 0.8 & $-4.1(-14.2$ to 6.0$)$ & 0.4 \\
\hline Corrected weekend midpoint & $-2.7(-5.6$ to 0.3$)$ & 0.07 & $-0.6(-4.1$ to 2.8$)$ & 0.7 & $-5.0(-15.3$ to 5.2$)$ & 0.3 \\
\hline
\end{tabular}

CI, confidence interval.

of circadian rhythm [22-24,35], and TRAF3IP1 (TRAF3 Interacting Protein 1), which influences, eg, neurogenesis and iris formation [36].

The G-allele of the rs372229746 was associated with earlier sleep midpoint at the age of eight years in weekdays and weekends. Although rs372229746 locates upstream to RAS p21 protein activator 4B (RASA4B) gene, the GWAS by Lane et al. has linked this variant with the FBXL13 (F-Box and Leucine Rich Repeat Protein 13) gene [21]. Notably, FBXL3 has been associated with the regulation of circadian rhythm [26-28] and a nearby genetic variant, rs3972456, which is also close to FBXL13 was found to be significantly associated with circadian preference also in the GWAS by Hu et al. [20]. Therefore, study here supports findings from all of these three GWASs [19-21] and other previous studies [26-28] reporting the association of FBXL13 with circadian rhythm.

The T-allele of the SNP rs9961653 was associated with earlier sleep midpoint at the age of eight years on weekends, as well as at the age of 17 years on weekdays. The weekend sleep midpoint is generally a better predictor of individual sleep rhythm than weekday midpoint of sleep, which is more influenced by the school or work schedule [37]. Moreover, rs9961653 is an intergenic variant with several nearby genes, such as RAX, CPLX4, and LMAN1 [19], of which RAX (retina and anterior neural fold homeobox) is involved with ocular development $[38,39]$.
As we have previously shown [17], self-reported circadian preference and objective sleep midpoint reflect on each other so that the late circadian preference in adolescence begins to manifest already in childhood as later sleep timing, becoming increasingly eveningoriented towards adolescence. Our observations in this longitudinal cohort are thus in line with previous studies showing a progressive shift to a later rhythm in adolescence [16]. In our previous study [17], the actual sleep timing grew increasingly different from age 8-17 years between individuals with morning and evening preferences, and here we show that the genetic effect becomes more apparent towards late adolescence. Thus, during childhood and early adolescence other factors, such as parental guidance regarding bedtimes [40,41], seem to have stronger effects on the circadian rhythm than genetic variants included in the PRS we studied.

\subsection{Strengths and limitations}

Strengths of our study include the assessment of sleep timing with objectively measured sleep midpoint, and the long duration of the follow-up period, from middle childhood to late adolescence, over a nine-year period. However, we had a limited sample size. In order to overcome this limitation, we used SNP-based PRSs. The use of PRSs limit the number of tests and have been shown to increase power in genomic studies. As a limitation, rs76899638 was not

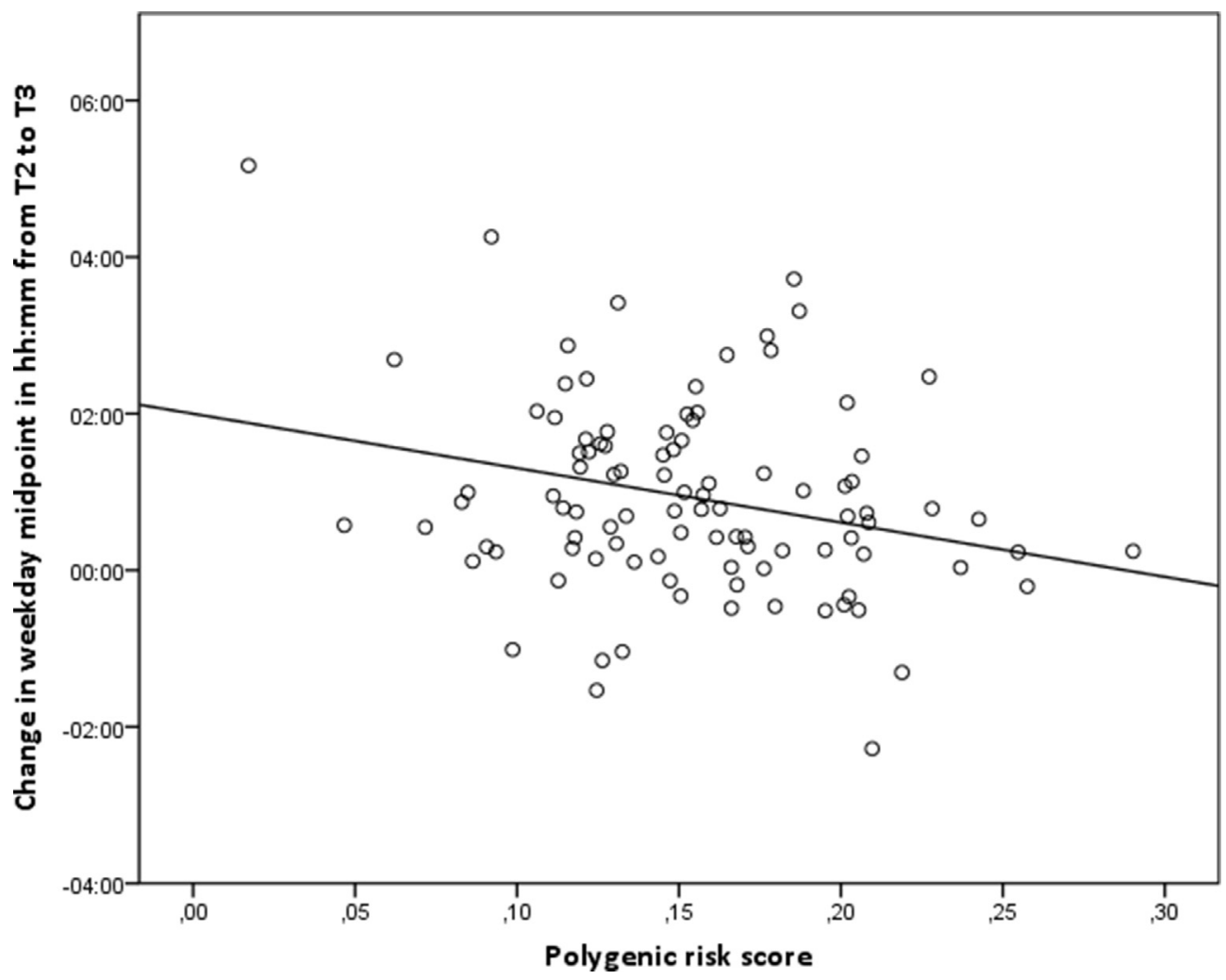

Fig. 2. The change in sleep midpoint during weekdays from the age of 12 years to the age of 17 years in relation to the polygenic risk score for morning preference. 
available in our sample. Also, rs516134 was excluded from the analyses as it had a MAF lower than five. Finally, the single MEQ item correlated only moderately with the full MEQ.

\subsection{Conclusion}

Our results show for the first time that genetic variants for circadian preference in adults is associated with objectively measured sleep timing trajectories from middle childhood to late adolescence, and also with the subjective circadian preference in late adolescence. In line with our assumption, those with a higher genetic tendency for morning preference showed an earlier grand average of sleep midpoints from middle childhood to late adolescence. In addition, the development of the circadian rhythm was moderated by the genetic risk score as the shift towards eveningness during the adolescence was significantly milder among individuals with a high PRS for morning preference. The genetic effect of these variants on sleep timing was stronger during late adolescence as compared to earlier developmental phases, suggesting that parental regulation of the sleep rhythm, probably being stronger at earlier ages, may overshadow the genetic component to some extent. The obtained information is novel and contributes to increasing the understanding of child and adolescent sleep timing and its challenges from a purely biological perspective.

\section{Acknowledgements}

The authors thank the Academy of Finland for funding this research.

\section{Conflicts of interest}

The authors have no conflicts of interest to disclose. This study was funded by the Academy of Finland.

The ICMJE Uniform Disclosure Form for Potential Conflicts of Interest associated with this article can be viewed by clicking on the following link: https://doi.org/10.1016/j.sleep.2018.04.015.

\section{References}

[1] Czeisler CA, Gooley J. Sleep and circadian rhythms in humans72. Cold Spring Harbor Laboratory Press; 2007. p. 579-97.

[2] Gau SS-F, Shang C-Y, Merikangas KR, et al. Association between morningnesseveningness and behavioral/emotional problems among adolescents. J Bio Rhythm 2007;22:268-74.

[3] Lange L, Randler C. Morningness-eveningness and behavioural problems in adolescents. Sleep Biol Rhythm 2011;9:12-8.

[4] Merikanto I, Pesonen A-K, Kuula L, et al. Eveningness as a risk for behavioral problems in late adolescence. Chronobiol Int 2017:1-10.

[5] Kitamura S, Hida A, Watanabe M, et al. Evening preference is related to the incidence of depressive states independent of sleep-wake conditions. Chronobiol Int 2010;27:1797-812.

[6] Merikanto I, Lahti T, Kronholm E, et al. Evening types are prone to depression. Chronobiol Int 2013;30:719-25. https://doi.org/10.3109/07420528.2013.784770.

[7] Merikanto I, Lahti T, Puolijoki $\mathrm{H}$, et al. Associations of chronotype and sleep with cardiovascular diseases and type 2 diabetes. Chronobiol Int 2013;30: 470-7. https://doi.org/10.3109/07420528.2012.741171.

[8] Merikanto I, Kronholm E, Peltonen M, et al. Circadian preference links to depression in general adult population. J Affect Disord 2015;188:143-8. https://doi.org/10.1016/j.jad.2015.08.061.

[9] Taillard J, Philip P, Chastang JF, et al. Is self-reported morbidity related to the circadian clock? J Biol Rhythm 2001;16:183-90. https://doi.org/10.1177/ 074873001129001764

[10] Merikanto I, Englund A, Kronholm E, et al. Evening chronotypes have the increased odds for bronchial asthma and nocturnal asthma. Chronobiol In 2014:31:95-101. https://doi.org/10.3109/07420528.2013.826672.

[11] Merikanto I, Lahti T, Seitsalo S, et al. Behavioral trait of morningnesseveningness in association with articular and spinal diseases in a population. PLoS One 2014:9:e114635.
[12] Merikanto I, Lahti T, Seitsalo S, et al. Eveningness has the increased odds for spinal diseases but the decreased odds for articular diseases with prospective hospital treatments. Biol Rhythm Res 2016:1-12.

[13] Adan A, Archer SN, Hidalgo MP, et al. Circadian typology: a comprehensive review. Chronobiol Int 2012;29:1153-75.

[14] Broms U, Pitkäniemi J, Bäckmand $\mathrm{H}$, et al. Long-term consistency of diurnaltype preferences among men. Chronobiol Int 2014;31:182-8.

[15] Roenneberg T, Kuehnle T, Juda M, et al. Epidemiology of the human circadian clock. Sleep Med Rev 2007:11:429-38.

[16] Roenneberg T, Kuehnle T, Pramstaller PP, et al. A marker for the end of adolescence. Curr Biol 2004;14:R1038-9.

[17] Kuula L, Pesonen AK, Merikanto I, et al. Development of late circadian preference: sleep timing from childhood to late adolescence. J Pediatr 2018 Mar;194:182-189.e1. https://doi.org/10.1016/j.jpeds.2017.10.068.

[18] Natale V, Di Milia L. Season of birth and morningness: comparison between the Northern and Southern hemispheres. Chronobiol Int 2011;28:727-30. https://doi.org/10.3109/07420528.2011.589934.

[19] Jones SE, Tyrrell J, Wood AR, et al. Genome-wide association analyses in 128,266 individuals identifies new morningness and sleep duration loci. PLoS Genet 2016;12:e1006125. https://doi.org/10.1371/journal.pgen.1006125.

[20] Hu Y, Shmygelska A, Tran D, et al. GWAS of 89,283 individuals identifies genetic variants associated with self-reporting of being a morning person. Nat Commun 2016;7:10448. https://doi.org/10.1038/ncomms10448.

[21] Lane JM, Vlasac I, Anderson SG, et al. Genome-wide association analysis identifies novel loci for chronotype in 100,420 individuals from the UK Biobank. Nat Commun 2016;7:10889. https://doi.org/10.1038/ncomms10889.

[22] Zheng B, Larkin DW, Albrecht U, et al. The mPer2 gene encodes a functiona component of the mammalian circadian clock. Nature 1999;400:169-73. https://doi.org/10.1038/22118.

[23] Zheng B, Albrecht U, Kaasik K, et al. Nonredundant roles of the mPer1 and mPer2 genes in the mammalian circadian clock. Cell 2001:105:683-94.

[24] Preitner N, Damiola F, Lopez-Molina L, et al. The orphan nuclear receptor REVERBalpha controls circadian transcription within the positive limb of the mammalian circadian oscillator. Cell 2002:110:251-60.

[25] Doi M, Ishida A, Miyake A, et al. Circadian regulation of intracellular G-protein signalling mediates intercellular synchrony and rhythmicity in the suprachiasmatic nucleus. Nat Commun 2011;2:327. https://doi.org/10.1038/ ncomms1316.

[26] Busino L, Bassermann F, Maiolica A, et al. SCFFbxl3 controls the oscillation of the circadian clock by directing the degradation of cryptochrome proteins. Science 2007;316:900-4. https://doi.org/10.1126/science.1141194.

[27] Godinho SIH, Maywood ES, Shaw L, et al. The After-Hours mutant reveals a role for Fbxl3 in determining mammalian circadian period. Science 2007;316: 897-900. https://doi.org/10.1126/science.1141138.

[28] Siepka SM, Yoo S-H, Park J, et al. Circadian mutant overtime reveals F-box protein FBXL3 regulation of cryptochrome and period gene expression. Cell 2007;129:1011-23. https://doi.org/10.1016/j.cell.2007.04.030.

[29] Dudbridge F. Power and predictive accuracy of polygenic risk scores. PLoS Genet 2013;9:e1003348. https://doi.org/10.1371/journal.pgen.1003348.

[30] Kuula L, Pesonen A-K, Kajantie E, et al. Sleep and lipid profile during transition from childhood to adolescence. J Pediatr 2016.

[31] Pesonen A-K, Martikainen S, Heinonen K, et al. Continuity and change in poor sleep from childhood to early adolescence. Sleep 2014;37:289.

[32] Raikkonen K, Matthews KA, Pesonen A-K, et al. Poor sleep and altered hypothalamic-pituitary-adrenocortical and sympatho-adrenal-medullary system activity in children. J Clin Endocrinol Metab 2010;95:2254-61.

[33] Hätönen T, Forsblom S, Kieseppä T, et al. Circadian phenotype in patients with the co-morbid alcohol use and bipolar disorders. Alcohol Alcohol 2008:43:564-8.

[34] Horne JA, Ostberg O. A self-assessment questionnaire to determine morningness-eveningness in human circadian rhythms. Int J Chronobiol 1975;4:97-110.

[35] Asher G, Gatfield D, Stratmann M, et al. SIRT1 regulates circadian clock gene expression through PER2 deacetylation. Cell 2008;134:317-28. https:// doi.org/10.1016/j.cell.2008.06.050.

[36] Larsson M, Duffy DL, Zhu G, et al. GWAS findings for human iris patterns: associations with variants in genes that influence normal neuronal pattern development. Am J Hum Genet 2011;89:334-43. https://doi.org/10.1016/ j.ajhg.2011.07.011.

[37] Roenneberg T, Wirz-Justice A, Merrow M. Life between clocks: daily temporal patterns of human chronotypes. J Biol Rhythm 2003;18:80-90. https:// doi.org/10.1177/0748730402239679.

[38] Mathers PH, Jamrich M. Regulation of eye formation by the Rx and pax6 homeobox genes. Cell Mol Life Sci CMLS 2000;57:186-94. https://doi.org/ $10.1007 /$ PLO0000683.

[39] Chassaing N, Vigouroux A, Calvas P. Mutations in the newly identified RAX regulatory sequence are not a frequent cause of micro/anophthalmia. Genet Test Mol Biomarkers 2009;13:289-90. https://doi.org/10.1089/gtmb.2008.0143.

[40] Crowley SJ, Acebo C, Carskadon MA. Sleep, circadian rhythms, and delayed phase in adolescence. Sleep Med 2007;8:602-12. https://doi.org/10.1016/ j.sleep.2006.12.002.

[41] Carskadon MA. Patterns of sleep and sleepiness in adolescents. Pediatrician $1990 ; 17: 5-12$ 